\title{
Peroxiredoxin 1 expression in active ulcerative colitis mucosa identified by proteome analysis and involvement of thioredoxin based on immunohistochemistry
}

\author{
KAYO HORIE $^{1}$, TETUO MIKAMI ${ }^{2}$, TSUTOMU YOSHIDA ${ }^{3}$, YUICHI SATO ${ }^{4}$ and ISAO OKAYASU ${ }^{3}$ \\ ${ }^{1}$ Department of Bioscience and Laboratory Medicine, Hirosaki University Graduate School of Health Sciences, \\ Hirosaki, Aomori 036-8564; ${ }^{2}$ Department of Pathology, Toho University School of Medicine, Ota-ku, \\ Tokyo 143-8540; ${ }^{3}$ Department of Pathology, Kitasato University School of Medicine Sagamihara, Yokohama, \\ Kanagawa 252-0374; ${ }^{4}$ Department of Molecular Diagnostics, Kitasato University School of \\ Allied Health Sciences, Sagamihara, Kanagawa 252-0373, Japan
}

Received August 31,2017; Accepted November 29, 2017

DOI: $10.3892 / \mathrm{ol} .2017 .7549$

\begin{abstract}
Ulcerative colitis (UC) is a chronic, relapsing, inflammatory bowel disease, and patients with long-standing $\mathrm{UC}$ are at high risk of developing colorectal cancer as a typical case of the organ-specific chronic inflammation-carcinoma sequence. Interactions between epithelial and stromal cells and alterations in a variety of stromal microenvironments have been demonstrated to have important roles in the carcinogenesis of UC-associated carcinoma. Therefore, the identification of proteins in the inflammatory microenvironment is important not only in the epithelium, however also in the stroma of UC inflammatory foci. To identify proteins associated with UC-associated carcinoma, the present study used proteomic analysis with two-dimensional electrophoresis and mass spectrometry. Differentially expressed proteins were assessed between active and inactive UC biopsy specimens. Results were verified by immunohistochemistry. Peroxiredoxin 1 (PRDX1) was among the proteins identified to have increased expression in active compared with inactive UC. Immunohistochemical analysis indicated that the expression of both PRDX1 and thioredoxin (TRX) increased with increasing inflammation grade in epithelial cells in UC mucosal crypts. PRDX1-positive stromal cells in the lower half of the lamina propria increased along with colitis severity. Furthermore, the expression of both PRDX1 and TRX proteins was increased in UC-associated neoplastic lesions compared with normal mucosa. A stepwise increase in PRDX1 expression was clear with increasing tumor
\end{abstract}

Correspondence to: Dr Kayo Horie, Department of Bioscience and Laboratory Medicine, Hirosaki University Graduate School of Health Sciences, 66-1 Honcho, Hirosaki, Aomori 036-8564, Japan E-mail: k-horie@hirosaki-u.ac.jp

Key words: ulcerative colitis, proteome analysis, oxidative stress, biomarker, peroxiredoxin 1 , thioredoxin progression in UC-associated tumorigenesis. Since PRDX1 and TRX overexpression was a unique characteristic of UC activity and UC-associated neoplastic lesions, PRDX1 and TRX expression may reflect oxidative stress along with the severity of colitis activity and UC-associated tumorigenesis in patients with UC.

\section{Introduction}

Chronic inflammation, driving carcinogenic pathways, increases the risk of developing cancer $(1,2)$, such as ulcerative colitis (UC)-associated colorectal cancer (3-6). UC features chronic, relapsing, and debilitating idiopathic inflammation of the colon mucosa and submucosa, and patients with long-standing UC are at high risk of neoplastic development (3). Endoscopy remains the gold-standard method for the detection and quantification of UC inflammation. UC-associated carcinomas are often difficult to detect endoscopically and to discriminate from inflammatory regenerative epithelium $(7,8)$. Inflammation is assessed by measuring $\mathrm{C}$-reactive protein (CRP) levels (9), erythrocyte sedimentation rate (10), interleukin-6 (IL-6) levels (11), and tumor necrosis factor alpha (TNF alpha) levels (12); these characteristics are all known as biomarkers of chronic inflammation in colon cancer. However, these inflammatory markers are not sufficient for estimating the risk of colon carcinoma (13).

Similarly, fecal calprotectin reflects neutrophil migration to the intestinal mucosa, which occurs during intestinal inflammation (14). Fecal calprotectin levels correlate with the severity of mucosal inflammation (15-18). However, it specificity is low in suspected inflammatory bowel disease (IBD) in children (19). Therefore, although a number of inflammatory markers are available, these are still being developed.

We previously reported that interactions between epithelial cells and the stromal microenvironment in UC may play an important role in the carcinogenic pathway $(3,20,21)$, suggesting the existence of a mechanism by which the interaction between epithelial cells and stromal cells leads to interstitial fibrosis. 
Thus, we sought to identify proteins that characterize the inflammatory microenvironment. In the present study, using proteomics, we identified proteins differentially expressed in active and inactive UC mucosal biopsies. Agarose two-dimensional gel electrophoresis (agarose-2DE) and matrix-assisted laser desorption ionization-time of flight/time of flight mass spectrometry (MALDI-TOF/TOF MS) identified several proteins possibly associated with the inflammatory colonic mucosa. Immunoreactive staining of histological sections of surgically removed samples using antibodies against these proteins revealed peroxiredoxin 1 (PRDX1) as a candidate protein overexpressed in active UC biopsy samples.

\section{Materials and methods}

Biopsy samples for proteomic analysis. The present study was approved by the Committee for Kitasato University Medical Ethics Organization (KMEO), and informed consent was obtained from all patients prior to the beginning of the study (KMEO B08-01 and KMEO B01-15).

Biopsy samples were obtained at Kitasato East University Hospital from two patients with active UC, two patients with inactive UC, and four unrelated normal healthy controls. Biopsies were taken from two sites in each case: The sigmoid colon and rectum. The UC activity was histologically determined by Matts score (22) of neighboring biopsy samples using the following criteria: Biopsy of an active UC of sigmoid colon, Matts 4; biopsy of an active UC of rectum, Matts 2; biopsy of an inactive UC of sigmoid colon and rectum, Matts 1 for both.

Histological examination of $U C$ regenerative mucosa and $U C$-associated neoplastic lesions. UC regenerative mucosa samples were collected from UC cases in the pathological collection of Kitasato University Hospital and Kitasato University East Hospital. The corresponding clinicopathological data are summarized in Table I.

UC-associated neoplastic lesion samples were previously described (21). Thirty-nine UC regenerative mucosae, six UC-associated low-grade dysplasias (LGD), five high-grade dysplasias (HGD), five UC-associated carcinomas (UCCA), and 22 normal control mucosae were collected.

Proteomic analysis. Protein extraction, agarose-2DE, in-gel digestion, and protein identification were performed as previously described $(23,24)$. Peptide mass fingerprinting (PMF) was performed using the Autoflex III MALDI-TOF/TOF MS (Bruker Corporation, Ettlingen, Germany). PMF and MS/MS spectra were submitted to MASCOT (http://www.matrixscience.com/search_from_select.html) for searching of the IPI human 20,081,114 database (74,049 sequences, 31,194,560 residues; www.ebi.ac.uk/IPI/Databases.html/) and identification of the corresponding proteins.

Western blot analysis. Western blot analysis was performed as previously described (21) using anti-PRDX1 (monoclonal, 1:1,000; Abnova, Taipei, Taiwan) and anti- $\beta$-actin (monoclonal, 1:10,000; Sigma-Aldrich; Merck KGaA, Darmstadt, Germany) antibodies. Proteins were extracted from sigmoid colon biopsy samples (two from active UC, two from inactive
UC, and three from normal controls). A UC-associated cancer cell line (UCCA-24) and a sporadic colorectal cancer cell line (KE43P) served as colon cancer controls (25).

Immunohistochemical staining. Immunohistochemical staining was performed as previously described (21) using anti-PRDX1 antibodies (monoclonal, 1:200; Abnova) and anti-TRX antibodies (polyclonal, 1:1,000; Abcam, Cambridge, MA, USA). Sections for TRX staining were incubated in $10 \mathrm{mM}$ citrate buffer (pH 6.0) and boiled in a microwave oven for $15 \mathrm{~min}$. After incubation with Protein Block Serum-Free (DakoCytomation, Glostrup, Denmark), sections were incubated overnight with anti-PRDX1 and anti-TRX antibodies at $4^{\circ} \mathrm{C}$.

The immunoreactivities of $\mathrm{UC}$ regenerative mucosal crypts were evaluated at the upper and lower halves independently. The staining intensities of PRDX1 in UC regenerative and normal mucosal epithelia were determined semi-quantitatively (none, 0 ; weak, 1; moderate, 2; intense, 3 ). The number of positive cells per $250-\mu \mathrm{m}$ mucosal length (mainly histiocytes) was counted in UC regenerative and normal mucosal stroma. TRX expression in UC regenerative and normal mucosal crypts was determined as the number of positive crypts/the total number of crypts. Immunoreactivity in UC-associated neoplastic lesions was evaluated by using a previously described scoring system (26), which combines staining intensity (none, 0; weak, 1; moderate, 2; intense, 3) with percentages of positive cells $(0,<1 \% ; 1,1-25 \% ; 2$, $25-50 \% ; 3,50-75 \% ; 4,>75 \%)$.

Statistical analysis. All statistical tests were conducted using StatView v5.0 (SAS Institute, Inc., Cary, NC, USA) or SPSS v16.0 for Windows (IBM, Inc., Chicago, IL, USA). For comparison of more than three groups, Kruskal-Wallis tests were performed with the Mann-Whitney $U$-test as a post-hoc test for comparisons between two groups. $\mathrm{P}<0.05$ was considered to indicate a statistically significant difference. The correlation between the Matts score and TRX protein expression was tested using Spearman's rank correlation coefficient.

\section{Results}

Proteomic analysis. Thirteen spots showed a more than 1.5-fold difference between active and inactive UC in agarose 2-DE and were digested with trypsin. MALDI-TOF/TOF MS identified nine proteins showing higher expression in active than in inactive UC and four proteins showing lower expression in active than in inactive UC; these results are shown in Table II.

PRDX1 expression was consistently higher in active than in inactive UC. PRDX1 spots from an active UC sigmoid colon biopsy showed 1.7-fold higher expression than those from an inactive UC sigmoid colon biopsy. A representative proteome map derived from the active UC sigmoid colon biopsy specimen is shown in Fig. 1A, while the relative ratio of PRDX1 spot volumes is shown in Fig. 1C.

Two electrophoretic spots of PRDX1 with different isoelectric points (PI) were identified using agarose-2DE. While both of these were confirmed to be PRDX1, the spot with a PI around 6 reflected methionine oxidation according to MALDI-TOF/TOF MS analysis. Thus, this spot represented the 
Table I. Clinicopathological data of the patients who provided UC regenerative specimens.

\begin{tabular}{lcccccc}
\hline & $\begin{array}{c}\text { Number } \\
\text { of cases }\end{array}$ & $\begin{array}{c}\text { Age (years) } \\
\text { (mean } \pm \text { s.d.) }\end{array}$ & $\begin{array}{c}\text { Gender } \\
\text { (M:F) }\end{array}$ & $\begin{array}{c}\text { Number } \\
\text { of samples }\end{array}$ & $\begin{array}{c}\text { Number of samples } \\
\text { classified by Matts score }\end{array}$ & $\begin{array}{c}\text { Duration from the } \\
\text { onset of UC (years mean } \pm \text { s.d.) }\end{array}$ \\
\hline Normal control & 22 & $61.0 \pm 13.7$ & $11: 11$ & 22 & & $12.4 \pm 1.3$ \\
UC cases & 35 & $41.8 \pm 15.2$ & $15: 20$ & 100 & 5 (Matts 1) & $7.0 \pm 4.7$ \\
& & & & & 22 (Matts 2) & $6.7 \pm 5.1$ \\
& & & & 55 (Matts 3) & $5.5 \pm 5.1$
\end{tabular}

M, male; F, female; s.d., standard deviation. Normal control mucosa specimens collected at least $5 \mathrm{~cm}$ from colon cancer lesions and not associated with IBD were also examined.

Table II. Proteins in active ulcerative colitis.

A, Upregulated proteins in active ulcerative colitis

Active/inactive fold

Spot no.

Protein name

\begin{tabular}{cc}
\hline Sigmoid & Rectum \\
\hline 2.0 & 1.6 \\
2.1 & 1.3 \\
2.0 & 1.5 \\
1.7 & 1.0 \\
1.5 & 1.6 \\
2.0 & 1.3 \\
2.5 & 2.4 \\
1.6 & 1.0 \\
1.6 & 1.1
\end{tabular}

$\mathrm{B}$, Downregulated proteins in active ulcerative colitis

Active/inactive fold

\begin{tabular}{llrr} 
Spot no. & Protein name & Sigmoid & Rectum \\
\hline 7 & Galectin-4 & 0.5 & 0.5 \\
1 & Cytokeratin 19 & 0.6 & 0.4 \\
6 & Carbonic anhydrase 1 & 0.4 & 0.4 \\
10 & Cytokeratin 19 & 0.3 & 0.5
\end{tabular}

Differentially expressed proteins of more than 1.5 fold or less than 0.66 fold between active and inactive ulcerative colitis are listed. The proteins were tryptic digested and analyzed with MALDI-TOF/TOF MS systems. Nine up regulated proteins and 4 down regulated proteins in active ulcerative colitis were identified.

oxidized form of PRDX1, while the spot with a PI of around 8 was the reduced form of PRDX1. The reduced PRDX1 spots were bigger and darker than the oxidized PRDX1 spots in active UC biopsy specimens. Conversely, oxidized PRDX1 expression was higher in inactive UC and normal biopsy specimens, as shown in Fig. 2.

Western blot analysis of PRDX1 expression. As shown in Fig. 3, PRDX1 expression was higher in active UC than in inactive
UC and normal colonic mucosae. In addition, PRDX1 was expressed in UCCA-24 and KE-43P colon cancer cell lines.

PRDX1 expression in UC regenerative mucosae. PRDX1 was expressed in the cytoplasm of epithelial cells. In stromal tissue, PRDX1 was mainly expressed in macrophages, as shown in Fig. 4. Fig. 5A shows PRDX1 immunohistochemical staining scores in UC regenerative and normal colonic mucosae. Both the upper and lower halves of the crypts showed increases in 

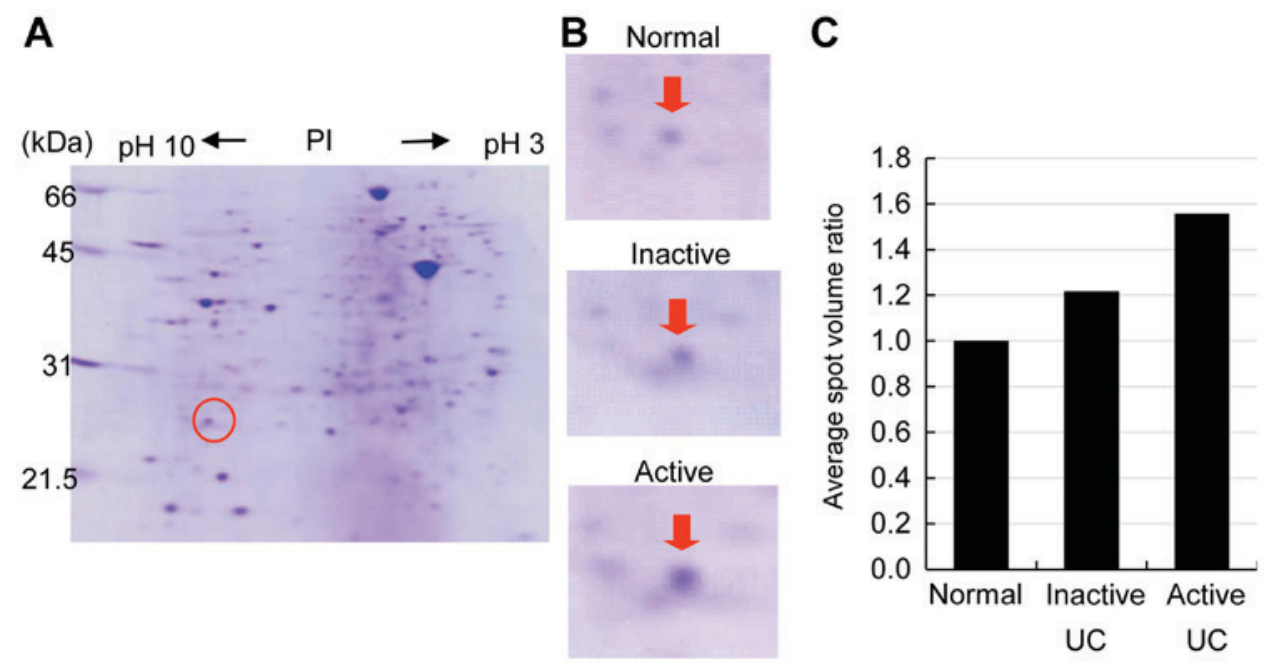

Figure 1. Agarose-2DE gel patterns of active ulcerative colitis sigmoid colon biopsies in Coomassie blue-stained two-dimensional electrophoresis gels. (A) Representative proteomic maps derived from active UC sigmoid colon biopsies. (B) Detailed gel images show PRDX1 expression in the normal mucosa, inactive UC, and active UC. (C) Average spot volume retio of PRDX1 in sigmoid colon biopsies. Active UC shows the highest PRDX1 expression compared with levels in inactive UC and normal mucosa. PI: Isoelectric point.

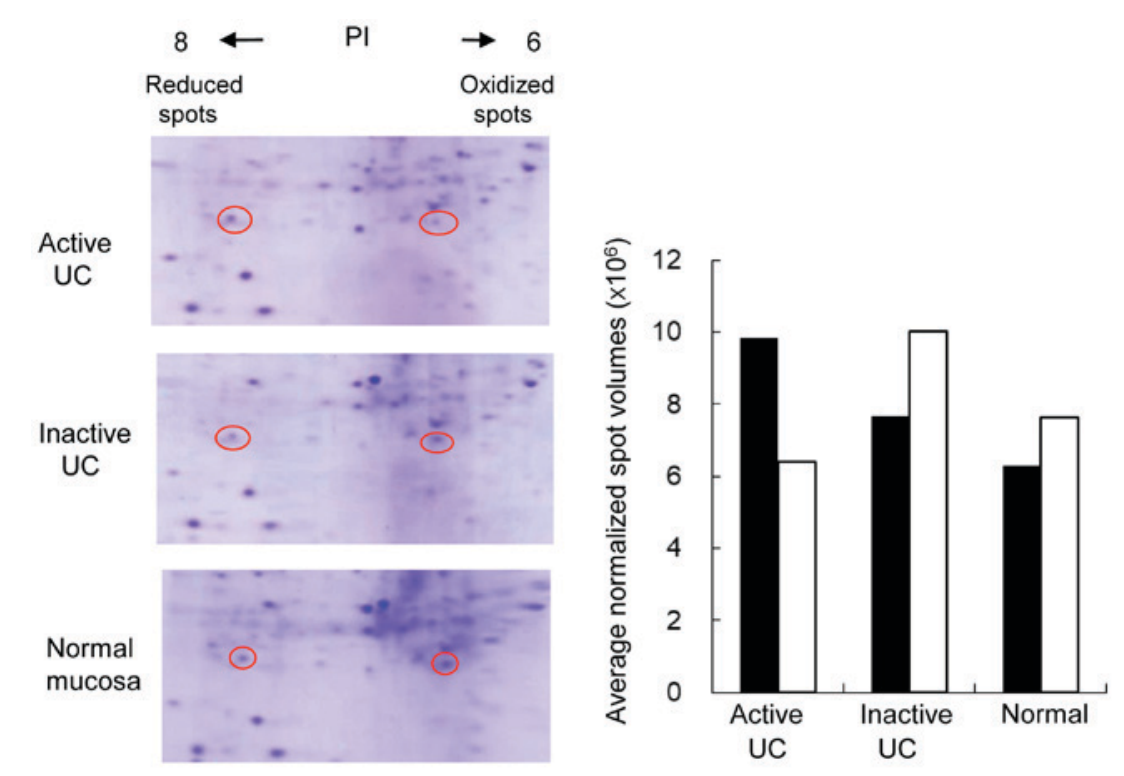

Figure 2. Isoelectric points of oxidized and reduced PRDX1 spots. The oxidized PRDX1 spots were compared with reduced PRDX1 spots using two-dimensional electrophoresis gels. Reduced PRDX1 spots were more highly expressed than oxidized PRDX1 spots in active UC biopsy specimens. Oxidized PRDX1 (open bars), reduced PRDX1 (black bars). PI: Isoelectric point.

PRDX1 expression with increasing inflammation levels as determined by Matts scores (Kruskal-Wallis test; $\mathrm{P}<0.05$ ). In particular, PRDX1 expression was significantly higher in active UC than in the normal mucosa (Matts 3: $\mathrm{P}<0.01$; Matts 4-5: P<0.001; Fig. 5A). In addition, Fig. 5B shows that in the lower half of the stroma, the active UC mucosa exhibited a significantly higher number of infiltrating PRDX1-positive cells than did the normal mucosa (Matts 4-5: $\mathrm{P}<0.001$ ). However, no significant difference was observed in the upper half of the stroma.

TRX expression in the UC-inflamed colonic mucosa. TRX expression was mainly observed in the cytoplasm of epithelial cells; however, some nuclear expression was also observed. TRX expression was evident in the cytoplasm of epithelial cells

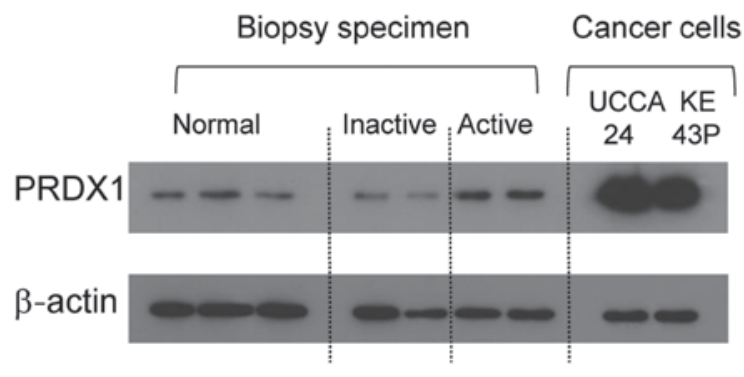

Figure 3. Western blot analysis of PRDX1 expression in protein extracts of sigmoid colon biopsy specimens (normal mucosa, inactive UC, and active UC) and colon cancer cell lysates. Two active UC samples showed higher PRDX1 expression than that in normal mucosa and inactive UC. Colon cancer cell lysates (UCCA-24: UC-associated colon cancer cells, KE43P: Sporadic colon cancer cells) also expressed PRDX1. $\beta$-actin expression was used as a control. 


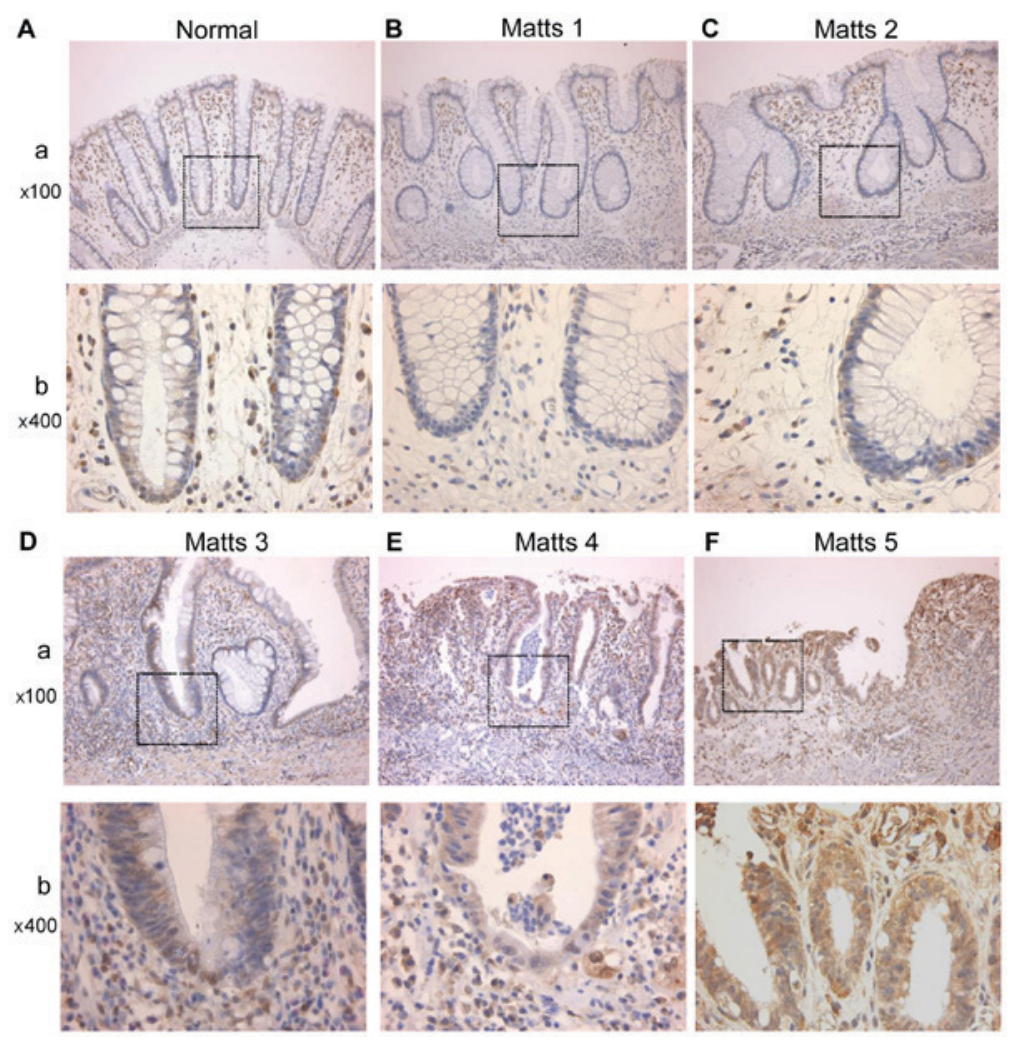

Figure 4. Immunohistochemical expression of PRDX1 along with Matts scores. (A) Normal, (B) Matts 1, (C) Matts 2, (D) Matts 3, (E) Matts 4, (F) Matts 5. (a) Low magnification (x100). (b) A higher magnification (x400) of the boxed area in panel (a) is shown.

A
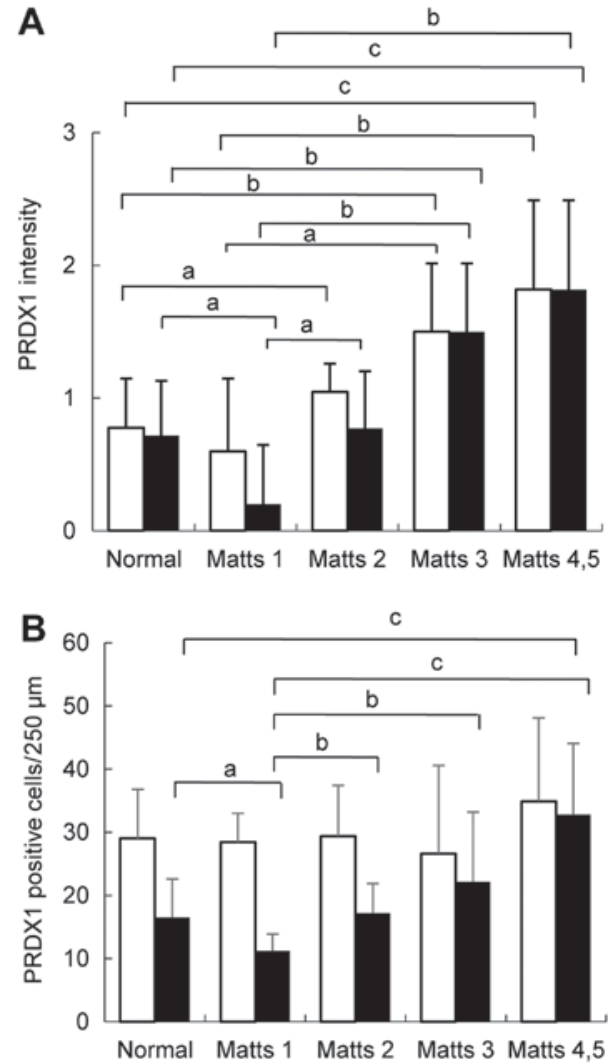

Figure 5. PRDX1 expression in UC regenerative mucosa and normal mucosa. (A) Staining intensity of PRDX1 in epithelial cells of four stages: 0, 1, 2, and 3. (B) PRDX1-positive cells per $250 \mu \mathrm{m}$ of stroma length were counted. Open bars, upper half of the crypt or lamina propria. black bars, lower half of the crypt or lamina propria. Data are presented as mean scores \pm standard deviations. ${ }^{\mathrm{a}} \mathrm{P}<0.05,{ }^{\mathrm{b}} \mathrm{P}<0.01$, and ${ }^{\mathrm{c}} \mathrm{P}<0.001$. in active UC (Matts 3 and 4-5), while very weak in the normal mucosae and inactive UC (Matts 1,2). TRX expression tended to increase gradually with increasing inflammation levels as determined by Matts score in both the upper and lower halves of the crypts, as shown in Fig. 6.

Crypts in which more than $50 \%$ of the epithelial cells expressed TRX were considered TRX-positive. Ratios of TRX-positive crypts (number of positive crypts/total number of crypts) are summarized in Table III. Ratios and Matts scores correlated in both the upper and lower halves of the crypts; $r=0.778(\mathrm{P}<0.001)$ for the upper half, and $r=0.821(\mathrm{P}<0.001)$ for the lower half.

PRDX1 expression in UC-associated dysplasia and carcinoma. Typical PRDX1 immunohistochemical staining patterns of UC-associated neoplastic lesions are shown in Fig. 7A and B. PRDX1 expression gradually increased from low-grade dysplasia to invasive carcinoma, as shown in Fig. 7C. LGD, HGD, and UCCA showed significantly higher PRDX1 expression than did the normal mucosa $(\mathrm{P}<0.01)$.

TRX expression in UC-associated dysplasia and carcinoma. Typical TRX immunohistochemical staining patterns of UC-associated neoplastic lesions are shown in Fig. 8A and B. TRX was mainly expressed in the cytoplasm, but also partially observed in the nucleus in UC-associated dysplasia and carcinoma. TRX expression in LGD, HGD, and UCCA was significantly higher than that in the normal mucosa $(\mathrm{P}<0.01)$, as shown in Fig. 8C. However, no significant difference was observed among the types of UC-associated neoplastic lesions (LGD, HGD, and UCCA). 
Table III. TRX expression in mucosal crypts of ulcerative colitis and normal colon.

\begin{tabular}{lllrrr}
\hline & Normal (\%) & Matts 1 $(\%)$ & Matts 2(\%) & Matts 3 (\%) & Matts 4,5 (\%) \\
\hline Upper half & $3 / 20(15.0)$ & $0 / 5(0)$ & $12 / 21(57.1)$ & $14 / 15(93.3)$ & $56 / 57^{\mathrm{a}}(98.2)$ \\
Lower half & $0 / 20(0)$ & $1 / 5(0.2)$ & $5 / 21(23.8)$ & $12 / 15(80.0)$ & $54 / 57^{\mathrm{b}}(94.7)$ \\
\hline
\end{tabular}

Data are shown as numbers of positive crypt/total numbers of crypt. Both upper and lower halves were separately examined. Both upper and lower halves showed positive correlation between TRX positive crypt ratio and Matts grade. Spearman's rank correlation coefficient: ${ }^{\mathrm{a}} \mathrm{r}=0.778$, $\mathrm{P}<0.001,{ }^{\mathrm{b}} \mathrm{r}=0.821, \mathrm{P}<0.001$.
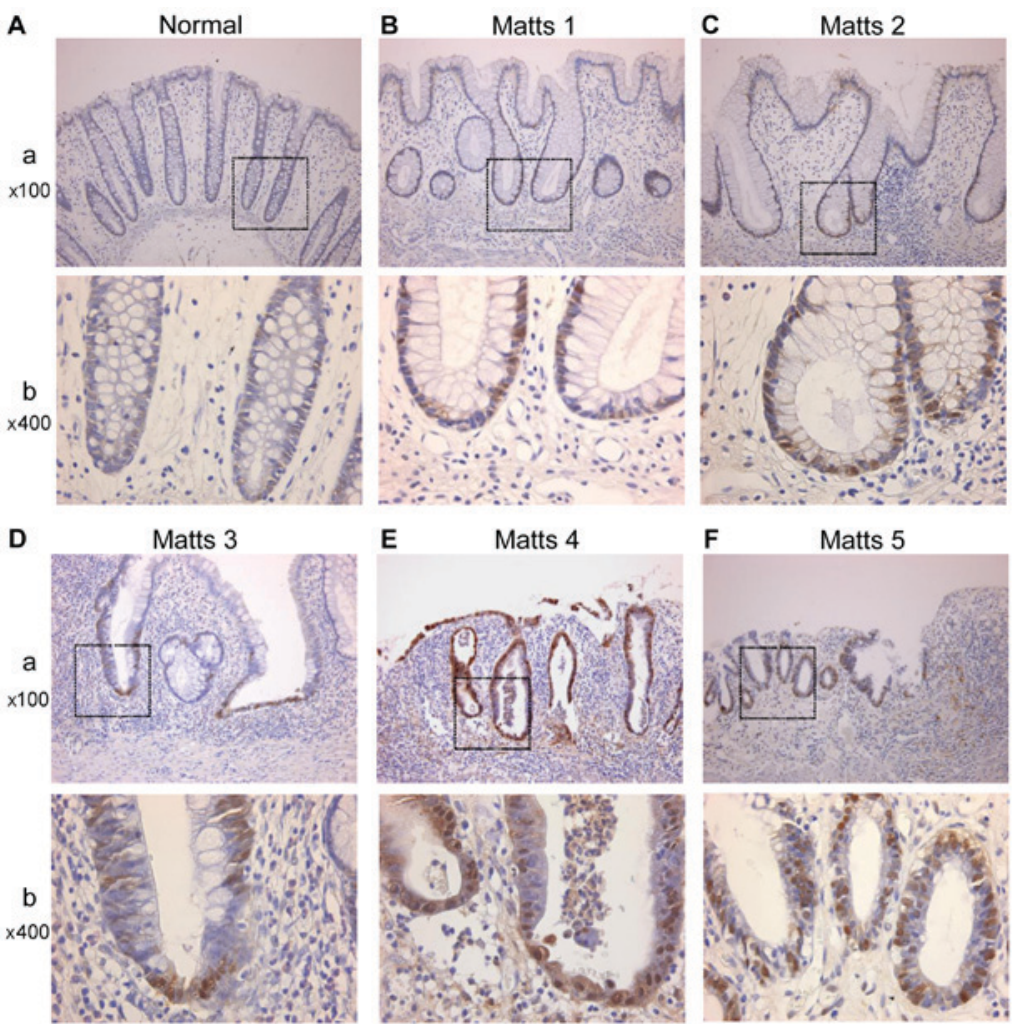

Figure 6. Representative TRX immunohistochemical staining patterns of UC regenerative mucosa by Matts classification. (A) Normal, (B) Matts 1, (C) Matts 2 , (D) Matts 3, (E) Matts 4, (F) Matts 5. (a) Low magnification (x100). (b) A higher magnification (x400) of the boxed area in panel (a) is shown.

\section{Discussion}

PRDXs are a class of thiol peroxidases that degrade hydroperoxides to water (27). PRDXs contain essential catalytic cysteine residues and are mainly reduced by thioredoxins (TRXs) (28). Mammals have six different PRDXs (PRDX1-6) (29). Various types of PRDXs have diverse and even opposing functions (30). PRDX1 is a versatile molecule regulating cell growth, differentiation, and apoptosis. PRDX1 is overexpressed in breast cancer (31), where it is significantly associated with tumor invasion, nodal metastasis, and advanced disease stage (32). Moreover, reduced PRDX1 expression is an important factor in esophageal squamous cancer progression (33).

Oxidative stress plays an important role in the carcinogenesis of various cancers (34). PRDX1 regulates ROS-dependent signaling pathways, which play important roles in the progression and metastasis of human tumors (35). However, the biological function of PRDX1 in chronic inflammation-induced carcinogenesis, such as in UC-associated carcinoma, remains unclear (36).

In the present study, active and inactive UC biopsy samples were compared using proteomics and immunohistochemistry. PRDX1 was identified as an upregulated protein in active UC specimens. PRDX1 expression increased with inflammation levels in epithelial cells in the crypts of the UC regenerative mucosa. Furthermore, PRDX1 was mainly expressed in macrophages in the stroma of the UC regenerative mucosa. In particular, PRDX1 expression was significantly higher in the lower half of the lamina propria from the active UC mucosa than that from the normal and inactive UC mucosae, suggesting its association with inflammation activity. A previous study observed that monocytes in the blood release PRDX1 under inflammatory conditions (37). Our results suggest that PRDX1 expression in the epithelium and stromal tissue protects against oxidative stress in inflammatory foci.

Currently, no reliable biomarker exists for monitoring disease activity in UC. CRP is often used as a biomarker of UC activity, 

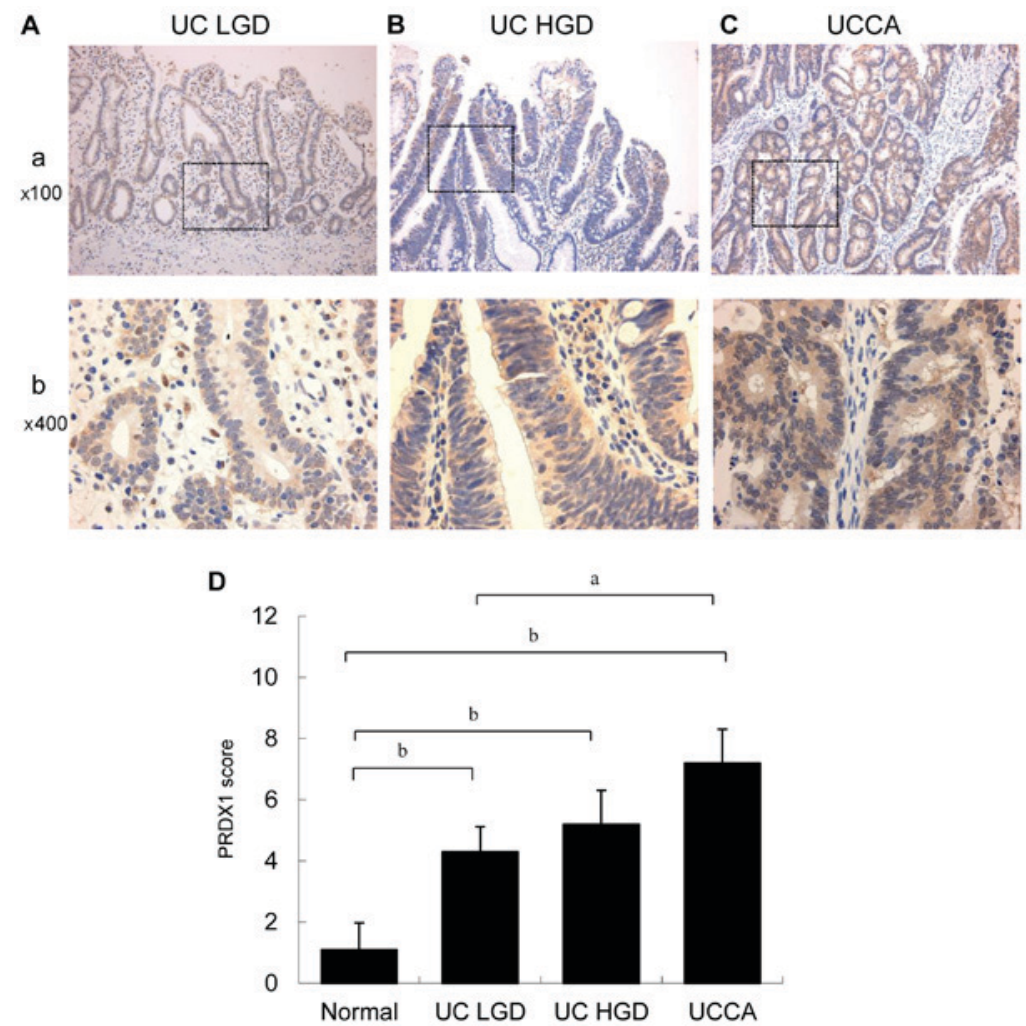

Figure 7. Immunohistochemical staining of PRDX1 in UC-associated neoplastic lesions. (A) UC LGD, UC low-grade dysplasia. (B) UC HGD, UC high-grade dysplasia. (C) UCCA, UC-associated carcinoma. (a) Low magnification (x100). (b) A higher magnification (x400) of the boxed area in panel (a) is shown. (D) The epithelial expression of PRDX1 in UC-associated neoplastic lesions. Data are shown as mean scores \pm standard deviations. ${ }^{a} \mathrm{P}<0.05$; ${ }^{\mathrm{b}} \mathrm{P}<0.01$.

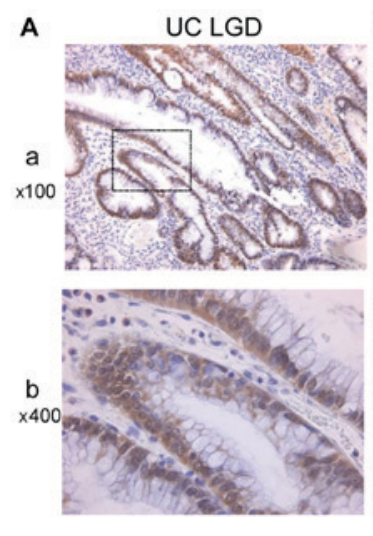

B UC HGD

C UCCA
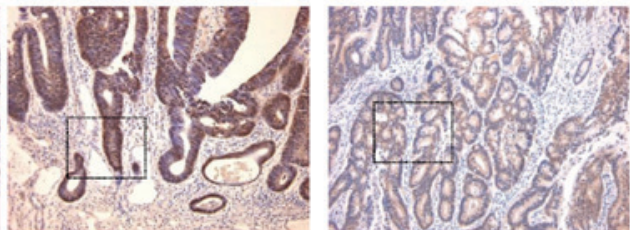

D
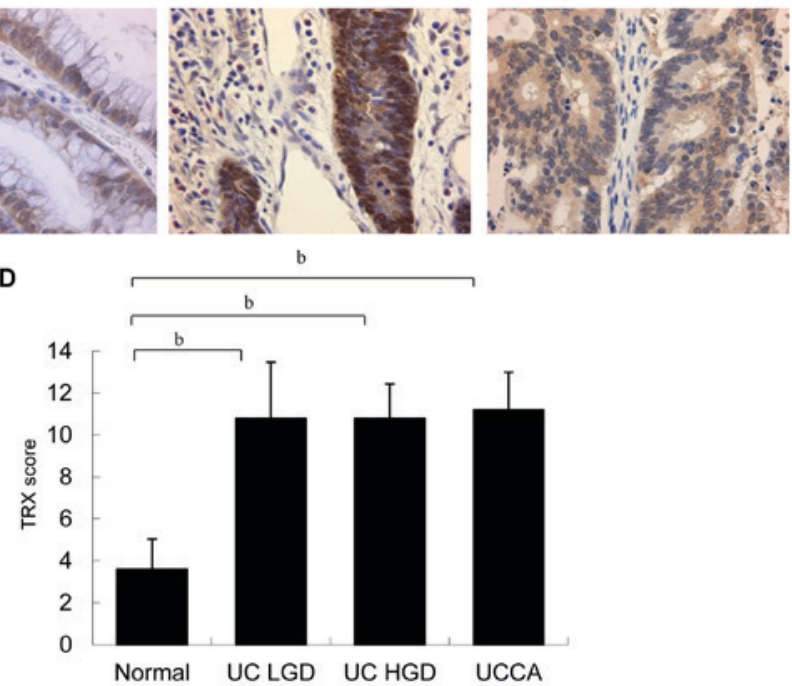

Figure 8. Immunohistochemical staining for TRX expression in UC-associated neoplastic lesions. (A) UC LGD, UC low-grade dysplasia. (B) UC HGD, UC high-grade dysplasia. (C) UCCA, UC-associated carcinoma. (a) Low magnification (x100). (b) A higher magnification (x400) of the boxed area in panel (a) is shown. (D) The epithelial expression of TRX in UC-associated neoplastic lesions. Data are shown as mean scores \pm standard deviations. ${ }^{\mathrm{b}} \mathrm{P}<0.01$.

but CRP levels are sometimes insufficient to reflect UC activity. Therefore, biomarkers of UC activity with sufficient sensitivity and specificity are desired. In recent studies, serum leucine-rich alpha-2-glycoprotein (LRG) concentrations correlated with 
UC activity. Increased LRG expression was detected in the cytoplasm of epithelial cells in inflamed tissues. Thus, inflamed colon tissue may be a potential source of increased serum LRG in patients with UC (38). In addition, other reports showed that urine levels of prostaglandin E-major urinary metabolite (PGE-MUM) were significantly correlated with active UC. Furthermore, compared with CRP levels, PGE-MUM demonstrated increased sensitivity for reflecting UC activity (39-41). However, these markers are still being developed. Our results showed that PRDX1 expression correlated with UC activity, suggesting its potential for monitoring oxidative stress in the colonic mucosa in association with UC activity.

In addition, we confirmed TRX expression in the UC regenerative mucosa. TRX is a redox-regulating protein involved in cellular redox homeostasis and cell survival. TRX is expressed at relatively high levels in several cancers (42). Our results showed that TRX expression increased according to the severity of inflammation in epithelial cells in the crypts of the UC regenerative mucosa. TRX expression correlated with UC activity, suggesting its potential as a biomarker for local oxidative stress related to UC activity.

We confirmed PRDX1 and TRX overexpression in UC-associated neoplastic lesions. PRDX1 expression was significantly higher in UC-associated neoplastic lesions than in the normal mucosa, and it increased gradually from LGD to invasive UCCA. Our results are consistent with an earlier report indicating that PRDX1-positivity scores were significantly higher in colorectal cancer than in the normal mucosa (36). TRX expression was also significantly higher in UC-associated neoplastic lesions than in the normal mucosa. In a previous study, TRX levels were elevated in cisplatin-resistant gastric and colon cancer cells (42). Our results showed that both PRDX1 and TRX proteins were highly expressed in UC-associated neoplastic lesions, and suggest that the proteins may indicate the presence of UCCA with oxidative stress. Since surveillance by endoscopic examination sometimes fails to detect cancer lesions (8), easily measurable biomarkers of UCCA are desired. Although it is necessary to confirm that PRDX1 and TRX are released from the UC regenerative mucosa and UCCA cells into the serum, measuring these proteins in serum samples may help the clinical detection of malignancy and inflammation activity in the future.

In this study, two electrophoretic spots of PRDX1 with different PIs were identified using agarose-2DE; the reduced form of PRDX1 was more highly expressed in active UC biopsy specimens, and the oxidized form was more highly expressed in inactive UC and normal biopsy specimens. Moreover, reduced PRDX1 was overexpressed in colon cancer cell lines (UCCA-24, KE-43P) (data not shown). Thus, PRDX1 expression and oxidative stress are related in UC inflammatory foci and colon cancer cell lines. PRDX1 expression should be considered not only in terms of increase/decrease, but also in terms of the kinetics of reduced/oxidized PRDX1, which can be visualized by agarose-2DE based on the PIs. As a preliminary study, we exposed UCCA 24 cells to $200 \mu \mathrm{M} \mathrm{H}_{2} \mathrm{O}_{2}$ for 20 min, which resulted in PRDX1 upregulation (data not shown). Exposure to $\mathrm{H}_{2} \mathrm{O}_{2}$ enhanced the expression levels of PRDX1-6 in human umbilical vein endothelial cells (43) and PRDX1 expression in normal human colon cells (36). PRDX1 and TRX expression suggests the presence of oxidative stress in the UC background mucosa, and these two proteins may be involved in UCCA carcinogenesis. Our study provides insights into carcinogenic pathways involving chronic inflammation in patients with UC.

In conclusion, PRDX1 and TRX are expressed in the UC inflamed mucosa and reflect the degree of inflammation. PRDX1 and TRX overexpression is a unique characteristic of UC-associated neoplastic lesions, including UCCA, and may reflect oxidative stress from inflammatory processes in UC. Further studies are warranted to identify the mechanisms underlying PRDX1 and TRX functions in UC-associated carcinogenesis with oxidative stress and to identify novel therapeutic targets for the treatment of UC-associated neoplastic lesions.

\section{Acknowledgements}

The authors would like to thank Dr. Ryo Nagashio for proteomic analysis support. This study was partially supported by a project grant for post-graduate students from Kitasato University Graduate School of Medical Sciences.

\section{References}

1. Coussens LM and Werb Z: Inflammation and cancer. Nature 420: 860-867, 2002.

2. Ohshima H, Tazawa H, Sylla BS and Sawa T: Prevention of human cancer by modulation of chronic inflammatory processes. Mutat Res 591: 110-122, 2005.

3. Okayasu I: Development of ulcerative colitis and its associated colorectal neoplasia as a model of the organ-specific chronic inflammation-carcinoma sequence. Pathol Int 62: 368-380, 2012.

4. Wong NA and Harrison DJ: Colorectal neoplasia in ulcerative colitis-recent advances. Histopathology 39: 221-234, 2001.

5. Okayasu I, Hana K, Yoshida T, Mikami T, Kanno J and Fujiwara M: Significant increase of colonic mutated crypts in ulcerative colitis correlatively with duration of illness. Cancer Res 62: 2236-2238, 2002.

6. Rhodes JM and Campbell BJ: Inflammation and colorectal cancer: Ibd-associated and sporadic cancer compared. Trends Mol Med 8: 10-16, 2002.

7. Sada M, Igarashi M, Yoshizawa S, Kobayashi K, Katsumata T, Saigenji K, Otani Y, Okayasu I and Mitomi H: Dye spraying and magnifying endoscopy for dysplasia and cancer surveillance in ulcerative colitis. Dis Colon Rectum 47: 1816-1823, 2004.

8. Fujii S, Katsumata D and Fujimori T: Limits of diagnosis and molecular markers for early detection of ulcerative colitis-associated colorectal neoplasia. Digestion 77 (Suppl 1): S2-S12, 2008.

9. Vermeire S, Van Assche G and Rutgeerts P: C-reactive protein as a marker for inflammatory bowel disease. Inflamm Bowel Dis 10: 661-665, 2004

10. Sachar DB, Smith H, Chan S, Cohen LB, Lichtiger S and Messer J: Erythrocytic sedimentation rate as a measure of clinical activity in inflammatory bowel disease. J Clin Gastroenterol 8: 647-650, 1986.

11. Gabay C: Interleukin-6 and chronic inflammation. Arthritis Res Ther 8 (Suppl 2): S3, 2006.

12. Braegger CP, Nicholls S, Murch SH, Stephens S and MacDonald TT: Tumour necrosis factor alpha in stool as a marker of intestinal inflammation. Lancet 339: 89-91, 1992.

13. Song M, Wu K, Ogino S, Fuchs CS, Giovannucci EL and Chan AT: A prospective study of plasma inflammatory markers and risk of colorectal cancer in men. Br J Cancer 108: 1891-1898, 2013.

14. Schoepfer AM, Beglinger C, Straumann A, Trummler M, Vavricka SR, Bruegger LE and Seibold F: Fecal calprotectin correlates more closely with the simple endoscopic score for crohn's disease (SES-CD) than CRP, blood leukocytes and the CDAI. Am J Gastroenterol 105: 162-169, 2010.

15. Gisbert JP and McNicholl AG: Questions and answers on the role of faecal calprotectin as a biological marker in inflammatory bowel disease. Dig Liver Dis 41: 56-66, 2009. 
16. Lehmann FS, Burri E and Beglinger $\mathrm{C}$ : The role and utility of faecal markers in inflammatory bowel disease. Therap Adv Gastroenterol 8: 23-36, 2015

17. Judd TA, Day AS, Lemberg DA, Turner D and Leach ST: Update of fecal markers of inflammation in inflammatory bowel disease. J Gastroenterol Hepatol 26: 1493-1499, 2011.

18. D'Haens G,Ferrante M, Vermeire S, Baert F, Noman M, Moortgat L, Geens P, Iwens D, Aerden I, Van Assche G, et al: Fecal calprotectin is a surrogate marker for endoscopic lesions in inflammatory bowel disease. Inflamm Bowel Dis 18: 2218-2224, 2012.

19. van Rheenen PF, Van de Vijver E and Fidler V: Faecal calprotectin for screening of patients with suspected inflammatory bowel disease: Diagnostic meta-analysis. BMJ 341: c3369, 2010.

20. Okayasu I, Yoshida T, Mikami T, Hana K, Yokozawa M, Araki K, Mitsuhashi J, Kikuchi M, Adachi E and Sada M: Mucosal remodeling in long-standing ulcerative colitis with colorectal neoplasia: Significant alterations of NCAM+ or alpha-SMA+ subepithelial myofibroblasts and interstitial cells. Pathol Int 59: 701-711, 2009.

21. Araki K, Mikami T, Yoshida T, Kikuchi M, Sato Y, Oh-Ishi M, Kodera Y, Maeda T and Okayasu I: High expression of hsp47 in ulcerative colitis-associated carcinomas: Proteomic approach. $\mathrm{Br}$ J Cancer 101: 492-497, 2009.

22. Matts SG: The value of rectal biopsy in the diagnosis of ulcerative colitis. Q J Med 30: 393-407, 1961.

23. Nagashio R, Sato Y, Jiang SX, Ryuge S, Kodera Y, Maeda T and Nakajima T: Detection of tumor-specific autoantibodies in sera of patients with lung cancer. Lung Cancer 62: 364-373, 2008.

24. Nagashio R, Sato Y, Matsumoto T, Kageyama T, Satoh Y, Ryuge S, Masuda N, Jiang SX and Okayasu I: Significant high expression of cytokeratins $7,8,18,19$ in pulmonary large cell neuroendocrine carcinomas, compared to small cell lung carcinomas. Pathol Int 60: 71-77, 2010.

25. Yamashita K, Yasuda S, Kuba T, Otani Y, Fujiwara M and Okayasu I: Unique characteristics of rectal carcinoma cell lines derived from invasive carcinomas in ulcerative colitis patients. Cancer Sci 95: 211-217, 2004.

26. Tokuyama W, Mikami T, Fujiwara M, Matsui T and Okayasu I: Midkine expression in colorectal tumors: Correlation with ki-67 labeling in sporadic, but not ulcerative colitis-associated ones. Pathol Int 57: 260-267, 2007.

27. Rhee SG and Woo HA: Multiple functions of peroxiredoxins: Peroxidases, sensors and regulators of the intracellular messenger $\mathrm{H}_{2} \mathrm{O}_{2}$ and protein chaperones. Antioxid Redox Signal 15: 781-794, 2011.

28. Karihtala P, Mantyniemi A, Kang SW, Kinnula VL and Soini Y: Peroxiredoxins in breast carcinoma. Clin Cancer Res 9: 3418-3424, 2003.

29. Poynton RA and Hampton MB: Peroxiredoxins as biomarkers of oxidative stress. Biochim Biophys Acta 1840: 906-912, 2014.

30. Zhang B, Wang Y and Su Y: Peroxiredoxins, a novel target in cancer radiotherapy. Cancer Lett 286: 154-160, 2009.
31. Cha MK, Suh KH and Kim IH: Overexpression of peroxiredoxin I and thioredoxin1 in human breast carcinoma. J Exp Clin Cancer Res 28: 93, 2009.

32. Zhou J, Shen W, He X, Qian J, Liu S and Yu G: Overexpression of prdx1 in hilar cholangiocarcinoma: A predictor for recurrence and prognosis. Int J Clin Exp Pathol 8: 9863-9874, 2015.

33. Hoshino I, Matsubara H, Akutsu Y, Nishimori T, Yoneyama Y, Murakami K, Sakata H, Matsushita K and Ochiai T: Tumor suppressor prdx1 is a prognostic factor in esophageal squamous cell carcinoma patients. Oncol Rep 18: 867-871, 2007.

34. Ambrosone CB: Oxidants and antioxidants in breast cancer. Antioxid Redox Signal 2: 903-917, 2000.

35. Ding C, Fan X and Wu G: Peroxiredoxin 1-an antioxidant enzyme in cancer. J Cell Mol Med 21: 193-202, 2017.

36. Chu G, Li J, Zhao Y, Liu N, Zhu X, Liu Q, Wei D and Gao C: Identification and verification of prdx 1 as an inflammation marker for colorectal cancer progression. Am J Transl Res 8: 842-859, 2016.

37. Liu CH, Kuo SW, Hsu LM, Huang SC, Wang CH, Tsai PR, Chen YS, Jou TS and Ko WJ: Peroxiredoxin 1 induces inflammatory cytokine response and predicts outcome of cardiogenic shock patients necessitating extracorporeal membrane oxygenation: An observational cohort study and translational approach. J Transl Med 14: 114, 2016

38. Serada S,Fujimoto M, Terabe F, Iijima H, Shinzaki S, MatsuzakiS, Ohkawara T, Nezu R, Nakajima S, Kobayashi T, et al: Serum leucine-rich alpha-2 glycoprotein is a disease activity biomarker in ulcerative colitis. Inflamm Bowel Dis 18: 2169-2179, 2012

39. Arai Y, Arihiro S, Matsuura T, Kato T, Matsuoka M, Saruta M, Mitsunaga M, Matsuura M, Fujiwara M, Okayasu I, et al: Prostaglandin e-major urinary metabolite as a reliable surrogate marker for mucosal inflammation in ulcerative colitis. Inflamm Bowel Dis 20: 1208-1216, 2014.

40. Arai Y, Matsuura T, Matsuura M, Fujiwara M, Okayasu I, Ito S and Arihiro S: Prostaglandin e-major urinary metabolite as a biomarker for inflammation in ulcerative colitis: Prostaglandins revisited. Digestion 93: 32-39, 2016.

41. Hagiwara SI, Okayasu I, Fujiwara M, Matsuura M, Ohnishi H, Ito $\mathrm{S}$, Kishimoto $\mathrm{H}$, Nambu $\mathrm{R}$ and Kagimoto $\mathrm{S}$ : Prostaglandin e-major urinary metabolite as a biomarker for pediatric ulcerative colitis activity. J Pediatr Gastroenterol Nutr 64: 955-961, 2017.

42. Marks PA: Thioredoxin in cancer-role of histone deacetylase inhibitors. Semin Cancer Biol 16: 436-443, 2006.

43. Mitsumoto A, Takanezawa Y, Okawa K, Iwamatsu A and Nakagawa Y: Variants of peroxiredoxins expression in response to hydroperoxide stress. Free Radic Biol Med 30: 625-635, 2001.

This work is licensed under a Creative Commons Attribution-NonCommercial-NoDerivatives 4.0 International (CC BY-NC-ND 4.0) License. 